\title{
Plasticidade da folha e lenho de cinco espécies lenhosas em duas áreas de restinga no Sul do Brasil
}

\author{
Maiara Matilde da Silva ${ }^{1,2} \&$ João Carlos Ferreira de Melo Júnior ${ }^{2}$
}

\author{
' Universidade Federal do Paraná, Programa de Pós-Graduação em Ecologia e Conservação \\ 2 Universidade da Região de Joinville, Programa Institucional de Pesquisa em Ciências Ambientais, \\ Laboratório de Morfologia e Ecologia Vegetal mmatilde.eco@gmail.com; jcmelo_wood@hotmail.com
}

Recebido em 30. VIII. 2016

Aceito em 25.VII.2017

DOI $10.21826 / 2446-8231201772204$

\begin{abstract}
RESUMO - O presente estudo verificou comparativamente a plasticidade de Clusia criuva Cambess., Myrsine venosa A. DC., Ocotea pulchella (Nees e Mart.) Mez, Pera glabrata (Schott) Poepp. Ex Baill e Ternstroemia brasiliensis Cambess. Foram mensurados atributos funcionais de folha e caule, e em campo foram tomados a altura e diâmetro caulinar à altura do peito. A heterogeneidade ambiental foi caracterizada pela análise química dos solos, espessura da serapilheira e umidade gravimétrica. Para cada atributo morfoanatômico calculou-se o índice de plasticidade fenotípica. Os atributos foliares apresentaram variação entre as restingas em todas as espécies estudadas, destacando-se a área específica foliar. Atributos da madeira pouco variaram, mostrando maior herdabilidade de caracteres ou condição hídrica similar do ponto de vista do uso do recurso hídrico. Os resultados corroboram com outros estudos e indicam que a folha é mais plástica, respondendo às variações do hábitat.
\end{abstract}

Palavras-chave: anatomia de madeira, morfologia foliar, respostas plásticas

ABSTRACT - Leaf and wood plasticity of five restinga species in two areas of the South Brazil. This study examined comparatively the plasticity of five "lenhosas" species occurring in two areas of salt marsh in Santa Catarina State, as follow: Clusia criuva Cambess., Myrsine venosa A. DC., Ocotea pulchella (Nees e Mart.) Mez, Pera glabrata (Schott) Poepp. Ex Baill and Ternstroemia brasiliensis Cambess. Functional attributes of leaf and stem were measured, and in the field trees' height and diameter were taken at breast height. The environmental heterogeneity was characterized by chemical analysis of the soil, thickness of litter and gravimetric moisture. For each morphological and anatomical attribute the phenotypic plasticity index was calculated. Leaf traits showed variation among the areas in all studied species, especially regarding specific leaf area. Wood attributes changed little, showing a higher inheritability of characters or similar responses to the water condition from the point of view of water resources' use of. The results corroborate to other studies indicating that leaf is more plastic to habitats variations.

Keywords: wood anatomy, leaf morphology, plastic response

\section{INTRODUÇÃO}

Restinga é um termo ecológico que se refere a um grande complexo fitogeográfico composto por um mosaico de formações vegetais (herbáceas, arbustivas e arbóreas) regidas por condições edáficas e que se distribui na planície arenosa de idade quaternária do litoral brasileiro (Araújo \& Lacerda 1987, Scarano 2002, Melo Júnior \& Boeger 2015). A restinga sul-brasileira está entre as latitudes $28^{\circ} 30^{\prime} \mathrm{S}$ no estado de Santa Catarina e $33^{\circ} 45^{\prime}$ 'S no extremo sul do Rio Grande do Sul (IBGE 2012).

De forma geral, plantas do ambiente de restinga estão expostas ao soterramento pela areia, à incidência frequente de ventos, à falta de água ou, em alguns locais, ao alagamento, à alta salinidade, à pobreza de nutrientes no solo e ao excesso de calor e luminosidade (Zamith \& Scarano 2004). Vários estudos têm demonstrado que a vegetação sujeita a esses fatores estressantes expressam respostas funcionais por meio de ajustes estruturais que contribuam efetivamente para seu crescimento e sobrevivência (Boeger \& Gluzezak 2006,
Rosado \& Mattos 2010, Melo Júnior \& Boeger 2016). A essas respostas é atribuído o conceito de plasticidade fenotípica, representando a capacidade de um mesmo genótipo produzir diferentes fenótipos para que o organismo possa ajustar seu desenvolvimento, fisiologia e história de vida em resposta às características do ambiente (Sultan 2000, Gratani 2014).

Estudos avaliando a plasticidade fenotípica têm sido desenvolvidos para determinar o melhor regime de luz para cultivo de espécies com importância econômica, como por exemplo o de Gondim et al. (2008), que demonstrou a variação anatômica de nas folhas de Colocasia esculenta L. (Schott), o taro japonês, sob diferentes intensidade de sombreamento; e o de Boeger et al. (2009), em que se analisou a estrutura foliar de quatro espécies medicinais consorciadas com Ilex paraguariensis (erva-mate), em três condições de luz, para contribuir para o delineamento de métodos de cultivo dessas espécies. Com o mesmo objetivo, de fornecer informações para manejo e cultivo, Lima et al. (2010) avaliaram o crescimento inicial e a plasticidade 
fenotípica de Caesalpinia echinata Lam (pau-brasil) - Fabaceae, Cariniana legalis (Martius) Kuntze (jequitibá) - Lecythidaceae e Genipa americana L. (Jenipapo) - Rubiaceae, em diferentes níveis de luz.

Frequentemente os estudos sobre plasticidade fenotípica são realizados com vistas a buscar a relação entre a variação morfoanatômica em espécies de comunidades naturais e a intensidade luminosa do ambiente, a taxa de herbivoria e o grau de sucessão (RéJorge 2007, Boeger et al. 2009, Anjos Silva 2010, Sabbi et al. 2010). No entanto, segundo Melo Júnior \& Boeger (2015), poucos estudos de plasticidade fenotípica têm relacionado as variações morfoanatômicas nas plantas com as características nutricionais do solo. Esses mesmos autores demonstraram que as espécies de restinga podem apresentar altos valores de plasticidade fenotípica, regidas principalmente pelas características edáficas do ambiente, que podem ser muitas vezes estressantes pela baixa disponibilidade de nutrientes e água. Todorovisky et al. (2015) comprovaram variações morfoanatômicas em Nectandra oppositifolia Ness. (Lauraceae) relacionadas às diferenças na quantidade de nutrientes nos solos de ambiente de restinga e floresta ombrófila densa.

Diante do exposto, o presente estudo verificou o plasticidade de cinco espécies lenhosas de restinga por meio da comparação de atributos foliares e do lenho de populações distribuídas em duas áreas de restinga arbustivo-arbórea do litoral de Santa Catarina caracterizadas por distintas condições edáficas. Sendo tais áreas relativamente próximas umas das outras, o que configura uma microescala espacial, este estudo teve como objetivos responder às seguintes questões: a) as espécies apresentam respostas estruturais semelhantes em ambas as áreas de ocorrência? b) quais fatores ambientais podem estar agindo como filtro ambiental? c) em qual área as espécies estão sob maior pressão ambiental e como as características morfoanatômicas refletem adaptações a esse ambiente?

\section{MATERIAL E MÉTODOS}

O estudo foi realizado em duas áreas de restinga da planície costeira do estado de Santa Catarina, litoral sul do Brasil, nos municípios de Balneário Barra do Sul e São Francisco do Sul. O primeiro localiza-se no litoral norte do estado ( $26^{\circ} 30^{\prime} \mathrm{S}$ e $\left.40^{\circ} 37^{\prime} \mathrm{W}\right)$, com $12 \mathrm{~km}$ de praia. Possui clima subtropical, com temperatura média anual de $23^{\circ} \mathrm{C}$ e precipitação média anual de $1.759 \mathrm{~mm} /$ ano (Knie 2002, Climate-Data 2014). São Francisco do Sul $\left(26^{\circ} 17^{\prime} \mathrm{S}\right.$ e $\left.48^{\circ} 33^{\prime} \mathrm{W}\right)$ apresenta clima influenciado pela umidade marítima, com temperatura média anual de $20,3^{\circ} \mathrm{C}$ e precipitação média de $1.874 \mathrm{~mm} /$ ano (Knie 2002). Ambas as áreas têm a vegetação de restinga distribuída em comunidades estruturalmente distintas, sendo a formação arbustivo-arbórea selecionada como área de coleta das espécies.
Realizou-se a caracterização ambiental das duas áreas de restinga com base nas características nutricionais do solo, espessura da serapilheira e umidade gravimétrica (Santos et al. 2013). A espessura da camada de serapilheira foi medida e anotada em campo, tomando-se 15 medidas por área. A umidade gravimétrica dos solos foi obtida pela diferença entre peso fresco e peso após secagem por $72 \mathrm{~h}$ em estufa a $105^{\circ} \mathrm{C}$ (Santos et al. 2013). Todas as coletas para a caracterização das áreas foram feitas na proximidade dos indivíduos amostrais.

As espécies Clusia criuva Cambess. (Clusiaceae), Myrcine venosa A. DC. (Myrsinaceae), Ocotea pulchella (Nees e Mart.) Mez (Lauraceae), Pera glabrata (Schott) Poepp. ex Baill (Peraceae) e Ternstroemia brasiliensis Cambess. (Pentaphylacaceae) foram selecionadas em função de serem apontadas como espécies abundantes nesse ambiente. Com posição social de destaque na estrutura da comunidade, juntas correspondem a 50\% da somatória do valor de importância das espécies da formação arbustivoarbórea de restinga (Melo Júnior \& Boeger 2015). Em cada área de estudo amostraram-se cinco espécimes de cada espécie selecionada, totalizando um grupo amostral de 10 plantas por espécie. De cada indivíduo, foram coletadas 25 folhas expostas ao sol, completamente expandidas, desprovidas de lesões e fixadas no $3^{\circ}$ e $4^{\circ}$ nós contando do ápice dos ramos. Amostras de madeira foram retiradas à altura do peito (1,3 $\mathrm{m}$ do solo) do ramo principal dos indivíduos por método não destrutivo (Barros et al. 2001). As amostras de lenho estão depositadas na Xiloteca Joiw, na Universidade da Região de Joinville. A biometria da altura $(\mathrm{m})$ e o diâmetro à altura do peito $(\mathrm{cm})$ dos espécimes coletados foram tomados em campo.

As folhas coletadas foram prensadas e secas em estufa a $60^{\circ} \mathrm{C}$ para posterior avaliação morfológica. Avaliaram-se os seguintes atributos funcionais foliares: massa seca foliar $(\mathrm{g})$; área foliar $\left(\mathrm{cm}^{2}\right)$, por meio de imagem digitalizada em scanner de mesa acoplado ao software Sigma Scan Pro (versão 5.0, SPSS Inc., Chicago IL, USA); área específica foliar (área foliar/massa seca foliar) $\left(\mathrm{cm}^{2} . \mathrm{g}-{ }^{1}\right)$ (Witkowski \& Lamont 1991); comprimento e largura do limbo foliar $(\mathrm{cm})$, medidos com auxílio de paquímetro digital Mitotoyo. As amostras de madeira foram amolecidas por cozimento, e cortes anatômicos nos planos tranversal, longitudinal e radial foram seccionados em micrótomo de deslize para a preparação de lâminas histológicas (Johansen 1940). Os cortes foram clarificados, corados com safranina, desidratados em série álcool-etílica crescente e montados em resina sintética (Kraus \& Arduim 1977, Paiva et al. 2006). Realizou-se a dissociação dos elementos celulares segundo o método de Franklin (1945), e estes foram corados segundo Berlyn \& Miksche (1976). A caracterização microscópica de lenho consistiu em avaliação quantitativa de características tais como: comprimento, diâmetro tangencial e frequência de elementos de vaso, conforme as orientações do IAWA Committee (1989).

As variáveis avaliadas apresentaram distribuição normal. Efetuou-se a comparação de médias entre as 
duas populações por meio do teste t de Student, com nível de significância de 5\% (Zar 1999), em software R Studio (Crawley 2007). Para cada variável morfológica e anatômica das espécies estudadas calculou-se o índice de plasticidade fenotípica (IPF) (Valladares et al. 2006).

\section{RESULTADOS E DISCUSSÃO}

\section{Condições edáficas da formação de restinga arbustivo- arbórea de Barra do Sul e São Francisco do Sul}

Os atributos nutricionais dos solos de restinga variaram entre as áreas estudadas (Tab. 1). O solo da restinga de Barra do Sul apresenta acidez média, com $\mathrm{pH}$ de 5,5, diferentemente do solo de São Francisco do Sul, que possui acidez muito alta, com $\mathrm{pH}$ de 3,53. Os elementos que mais variaram entre os locais estudados foram os íons $\mathrm{H}+\mathrm{Al}$ e $\mathrm{Ca}$. Em região sob condições tropicais, a elevada precipitação pluviométrica provoca uma intensa lixiviação de elementos químicos do

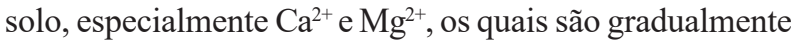
substituídos por $\mathrm{H}$ e $\mathrm{Al}$, o que intensifica a acidificação do solo (EMBRAPA 1997). Na restinga de São Francisco do Sul, a decomposição da matéria orgânica (MO) pode ser um dos principais processos de adição de íons $\mathrm{de} \mathrm{H}+$ ao solo por meio da formação de compostos orgânicos saturados de $\mathrm{H}+$, os quais atuam no aumento da acidez do solo (Amaral Filho et al. 2005, Faquin 2005).

Em solo com $\mathrm{pH}$ menores de 5,5 pode ocorrer alta toxicidade por $\mathrm{Al}$, afetando o crescimento e o desenvolvimento das plantas (EMBRAPA 1997, Miguel et al. 2010). Isso pode explicar o fato de os indivíduos amostrados em São Francisco do Sul serem, de forma geral, menores em altura e com caules com menor diâmetro. As altas concentrações de alumínio no solo de São Francisco do Sul podem ainda estar reduzindo a disponibilidade e absorção de P do solo e também na inibição competitiva da absorção de nutrientes catiônicos, como $\mathrm{Mg}^{2+}$ $\mathrm{e} \mathrm{K}^{+}$e micronutrientes (Faquin 2005), enquanto os menores teores de $\mathrm{Al}$ favorecem um aumento da disponibilidade de fósforo (EMBRAPA 1997). Os dados obtidos no presente estudo corroboram com tais observações, à medida que o solo de São Francisco do Sul apresenta maior concentração de $\mathrm{H}+\mathrm{Al}$ e uma concentração reduzida de magnésio e fósforo, enquanto em Barra do Sul o solo tem menores concentrações de $\mathrm{H}+\mathrm{Al}$ e maiores concentrações de $\mathrm{P}$ em comparação a São Francisco do Sul.

A concentração de Ca no solo demonstrou variação entre as duas áreas, mas em ambos os ambientes o teor de cálcio no solo é baixo, podendo ser maior em outras fisionomias de restinga desses locais (Melo Júnior \& Boeger 2015). Segundo White (1998), o cálcio geralmente se encontra em baixa concentração nos solos ácidos, que são típicos do território brasileiro.

Um elemento que apresentou variação acentuada entre as áreas de estudo foi o fósforo $(\mathrm{P})$, sendo a maior concentração no solo da restinga de Barra do Sul. O P é rapidamente adsorvido nas superfícies dos coloides do solo ou é precipitado como fosfatos de cálcio $(\mathrm{Ca})$, magnésio $(\mathrm{Mg})$, ferro $(\mathrm{Fe})$ e alumínio $(\mathrm{Al})$. Por isso, geralmente sintomas
Tabela 1. Valores médios dos atributos nutricionais dos solos $(n=15)$ e umidade gravimétrica $(n=15)$ nas restingas de São Francisco do Sul (SFS) Barra do $\mathrm{Sul}(\mathrm{BBS}) . \mathrm{H}+\mathrm{Al}=$ acidez potencial, $\mathrm{CTC}=$ capacidade de troca catiônica, $\mathrm{V}=$ saturação por bases, $\mathrm{MO}=$ matéria orgânica.

\begin{tabular}{lcc}
\hline Atributos do solo & SFS & BBS \\
\hline $\mathrm{pH}$ & 3,53 & 5,5 \\
$\mathrm{P}\left(\mathrm{mg} / \mathrm{dm}^{3}\right)$ & 1,00 & 3,7 \\
$\mathrm{~K}\left(\mathrm{cmolc} / \mathrm{dm}^{3}\right)$ & 1,30 & 0,11 \\
$\mathrm{Ca}\left(\mathrm{cmolc} / \mathrm{dm}^{3}\right)$ & 5,67 & 1,1 \\
$\mathrm{Mg}\left(\mathrm{cmolc} / \mathrm{dm}^{3}\right)$ & 1,00 & 1,00 \\
$\mathrm{H}+\mathrm{Al}\left(\mathrm{cmolc} / \mathrm{dm}^{3}\right)$ & 21,67 & 1,8 \\
$\mathrm{SB}\left(\mathrm{cmolc} / \mathrm{dm}^{3}\right)$ & 8,80 & 2,25 \\
$\mathrm{CTC}\left(\mathrm{cmolc} / \mathrm{dm}^{3}\right)$ & 30,47 & 4,05 \\
$\mathrm{~V}(\%)$ & 29,00 & 55,44 \\
$\mathrm{MO}\left(\mathrm{g} / \mathrm{dm}^{3}\right)$ & 14,67 & 9,0 \\
Espessura da serapilheira $(\mathrm{cm})$ & 3,70 & 2,69 \\
Umidade gravimétrica $(\mathrm{g})$ & 14,10 & 6,53 \\
\hline
\end{tabular}

de deficiência de $\mathrm{P}$ incluem a diminuição na altura da planta (Ronquim 2010), o que foi observado nos indivíduos amostrais da restinga de São Francisco do Sul.

O atributo edáfico que mais variou entre as áreas estudadas foi a capacidade de troca catiônica (CTC), sendo o maior valor obtido para o solo da restinga de São Francisco do Sul. Essa característica está diretamente relacionada ao conteúdo de matéria orgânica no solo. A CTC de um solo representa a quantidade de cátions permutáveis retidos na superfície dos coloides $\left(\mathrm{Ca}^{2+}+\mathrm{Mg}^{2+}+\mathrm{K}++\mathrm{H}^{+}+\mathrm{Al}^{3+}\right)$ (Lopes \& Guidolin 1989). Os coloides orgânicos constituem o húmus, produtos da decomposição da matéria orgânica, transformados biologicamente (Ronquim 2010). Na maioria dos solos tropicais prevalecem coloides com carga negativa e que, portanto, atraem cátions (SBCS 2004). Quanto maior a CTC do solo, maior o número de cátions que esse solo pode reter. Portanto, a CTC é uma característica físico-química fundamental da nutrição e da fertilidade do solo (Noble et al. 2000, Ronquim 2010). Conforme Ronquim (2010), se a maior parte da CTC do solo está ocupada por $\mathrm{Ca}^{2+} \mathrm{Mg}^{2+} \mathrm{e} \mathrm{K}^{+}$, pode-se dizer que se trata de um solo com alto potencial de nutrição vegetal, mas, se grande parte da CTC está ocupada por cátions potencialmente tóxicos como $\mathrm{H}^{+} \mathrm{e} \mathrm{Al}^{3+}$, esse solo é pobre. Um solo pode apresentar alto valor de CTC total, porém, caso uma parcela significativa das cargas negativas do solo (coloides) esteja adsorvendo íons $\mathrm{H}^{+}$, a CTC efetiva será menor (SBCS 2004). Nesse sentido, o solo de São Francisco do Sul pode apresentar maior toxidez às plantas em virtude da alta concentração de $\mathrm{Al}$, podendo ser menos fértil que o de Barra do Sul.

$\mathrm{O}$ índice de saturação por base (V) do solo da restinga de São Francisco do Sul $(29,00)$ foi menor que em Barra do $\mathrm{Sul}(55,44)$, constituindo assim um solo distrófico $(\mathrm{V}<50 \%)$. Em Barra do Sul, para esse atributo classifica-se o solo como eutrófico ( $\mathrm{V}>50 \%)$. A eutrofização é gerada principalmente por altos valores de $\mathrm{Na}$, provenientes em grande parte por spray marinho (Leão \& Dominguez 2000). Um índice V baixo significa que há muitos coloides sendo neutralizados por $\mathrm{H}^{+} \mathrm{eAl}^{3+}$, assim, o solo torna-se ácido, podendo até conter 
alumínio em nível tóxico às plantas (Lopes \& Guidolin 1989, Ronquim 2010), o que pode justificar a menor altura dos indivíduos de São Francisco do Sul.

Os óxidos de ferro e alumínio são importantes componentes da fração mineral dos solos sob condições tropicais e contribuem pouco para a capacidade de troca de cátions. Todavia a matéria orgânica (MO) desempenha um importante papel nas características químicas, físicas e microbiológicas do solo, sendo responsável por mais de $70 \%$ da capacidade de troca catiônica (CTC) dos solos tropicais, pelo fato de estar em sua maioria com cargas negativas (Ronquim 2010). A concentração de matéria orgânica (MO) no solo também resulta do acúmulo de serapilheira sobre o solo, que é maior em São Francisco do Sul.

A umidade gravimétrica foi mais elevada no solo de São Francisco do Sul. Esse atributo, que traduz a disponibilidade de água entre as partículas de solo, apresenta influência nos processos de evolução do solo (compactação do solo e aeração do solo) e no desenvolvimento das plantas (desenvolvimento radicular) (Timm et al. 2006). Embora a maior quantidade de água ocorra no solo de São Francisco do Sul, as concentrações de $\mathrm{Ca}$ e $\mathrm{Al}$ podem dificultar a absorção pelas plantas (Melo Júnior \& Boeger 2015).

\section{Variações estruturais das espécies}

Os atributos anatômicos do lenho e morfológicos foliares avaliados estão sumarizados na tabela 2. A espécie P. glabrata apresentou diferenças significativas em todos os atributos foliares entre os locais de estudo.

Em C. criuva e O. pulchella a massa seca foliar não evidenciou diferença estatística entre os espécimes das áreas estudadas. Em M. venosa e P. glabrata o maior valor de massa seca foliar ocorreu em Barra do Sul; isso pode ocorrer por causa da condição favorável do solo, que não é ácido e não contém tanto alumínio quando comparado com o solo de São Francisco do Sul (Miguel et al. 2010). A maior presença de Ca no solo, como ocorre em São Francisco do Sul, pode interferir negativamente na produção de matéria seca foliar, por impedir a absorção de K pela inibição competitiva, uma vez que K é um nutriente importante para o metabolismo da planta e pode influenciar na produção de matéria seca (Veloso et al. 2001, Faquin 2005), embora baixas concentrações de $\mathrm{Ca}$ atuem como sinergéticos (Faquin 2005).

A espécie $P$. glabrata apresentou diferença estatística em comprimento e largura do limbo foliar, C. criuva e $M$. venosa variaram apenas em comprimento do limbo e T. brasiliensis mostrou diferença estatisticamente significativa apenas para largura do limbo foliar. Esses atributos resultam na área foliar (AF), a qual variou em P. glabrata, T. brasiliensis e $M$. venosa, com maiores valores médios na restinga de Barra do Sul, exceto em $T$. brasiliensis, que foi maior em São Francisco do Sul. De acordo com Melo Júnior \& Boeger (2015), a área foliar não possui relação com a diversidade funcional do ambiente de restinga, ou seja, a disponibilidade de nutrientes no solo do ambientais de restinga pode influenciar mais fortemente em outras características foliares mais importantes para a sobrevivência das plantas na restinga.

$\mathrm{O}$ atributo morfológico foliar que apresentou diferença estatisticamente significativa entre os indivíduos de todas as espécies analisadas foi a área específica foliar (AEF) (Tab. 2), sendo as maiores médias observadas nas plantas da restinga de Barra do Sul. A AEF expressa a razão entre área foliar e massa seca da folha, trata-se de um fator importante por descrever a alocação da biomassa da folha por unidade de área, refletindo o "trade-off " entre rápida produção de biomassa e eficiente conservação de nutrientes (Witkowski \& Lamont 1991, Poorter \& Garnier 1999). Maiores AEF demonstram que os indivíduos de Barra do Sul investem mais em produção fotossintética, enquanto menores AEF são características de plantas xeromórficas (Boeger et al. 2008). A xeromorfia é resultado do maior desenvolvimento de tecidos de sustentação mecânica e pode estar relacionada com a disponibilidade hídrica do ambiente (Melo Júnior et al. 2012).

Os atributos anatômicos de lenho não apresentaram diferenças estatísticas significativas (Tab. 2), exceto em $M$. venosa, que exibiu diferença para o atributo de frequência de vaso. É consenso que a evolução do lenho está diretamente relacionada à disponibilidade hídrica e ao desenvolvimento de estratégias para maximizar a condutividade hidráulica (Ennos 1999, Sperry 2003). Segundo Carlquist (1977), quanto maior o diâmetro dos vasos, maior a eficiência na condução de água, bem como a suscetibilidade de formação de bolhas de ar que acabam interrompendo o fluxo de água e sais minerais. Estudo realizado com Andira fraxinifolia Betnh. (Fabaceae) em ambiente de restinga com maior concentração de sódio no solo mostrou que o lenho dessa espécie é formado por vasos de grande diâmetro e baixa frequência por unidade de área (Silva et al. 2016). Por outro lado, a presença de vasos numerosos e estreitos contribui efetivamente para aumentar a segurança na condutividade hidráulica, pois, se ocorrerem embolias em alguns vasos, o fluxo de seiva é garantido pelos outros vasos encontrados em grande número (Baas \& Carlquist 1985, Denardi \& Marchiori 2005). Quanto menor for o calibre do elemento de vaso, maior será a sua frequência por unidade de área (Dickison 2000, Luchi 2004), o que foi observado no lenho das espécies P. glabrata e M. venosa neste estudo. Por outro lado, as demais espécies com populações amostradas na restinga de São Francisco do Sul apresentaram maior diâmetro acompanhado pela maior frequência de vasos. Tal combinação pode estar relacionada ao aumento na eficácia do transporte de água para as folhas (Baas et al. 1983).

\section{Potencial plástico das espécies}

O índice de plasticidade (Tab. 3), considerado alto quando $\geq 0,6$ (Valladares et al. 2006), corrobora com diversos estudos, o que demonstra que a folha é o órgão vegetal que mais responde às mudanças no ambiente $\mathrm{e}$ apresenta elevada plasticidade fenotípica (Dickison 2000, Boeger \& Gluzezak 2006, Silva et al. 2016, Melo Júnior $\&$ Boeger 2016). No presente estudo, os maiores valores de 
Tabela 2. Valores médios e respectivos desvios-padrão $( \pm)$ das variáveis morfoanatômicas de caule e folha das cinco espécies em cada restinga estudada. Letras diferentes na mesma linha indicam diferença estatisticamente significativa $(\mathrm{p}<0,05)$.

\begin{tabular}{|c|c|c|}
\hline Espécie / Variável & São Francisco do Sul & Balneário Barra do Sul \\
\hline \multicolumn{3}{|l|}{ Clusia criuva } \\
\hline Altura da planta (m) & $4.33 \pm 0.77 \mathrm{a}$ & $4.53 \pm 0.44 \mathrm{a}$ \\
\hline Diâmetro do caule $(\mathrm{cm})$ & $34.70 \pm 5.37 \mathrm{a}$ & $30.20 \pm 2.20 \mathrm{~b}$ \\
\hline Comprimento do elemento de vaso (mm) & $848.32 \pm 104.59 \mathrm{a}$ & $800.52 \pm 95.45 \mathrm{a}$ \\
\hline Diâmetro tangencial do vaso $(\mathrm{mm})$ & $100.80 \pm 22.10 \mathrm{a}$ & $75.33 \pm 16.79 \mathrm{a}$ \\
\hline Frequência de vasos $\left(\mathrm{n} .{ }^{\circ} / \mathrm{mm}^{2}\right)$ & $13.99 \pm 3.14 \mathrm{a}$ & $12.87 \pm 2.63 \mathrm{a}$ \\
\hline Comprimento foliar (mm) & $100.06 \pm 14.25 \mathrm{~b}$ & $116.43 \pm 16.28 \mathrm{a}$ \\
\hline Largura foliar $(\mathrm{mm})$ & $49.11 \pm 10.62 \mathrm{a}$ & $44.81 \pm 7.89 \mathrm{a}$ \\
\hline Área foliar $\left(\mathrm{cm}^{2}\right)$ & $30.22 \pm 12.71 \mathrm{a}$ & $28.74 \pm 7.06 \mathrm{a}$ \\
\hline Área específica foliar $\left(\mathrm{cm}^{2} / \mathrm{g}\right)$ & $40.19 \pm 4.43 b$ & $49.76 \pm 7.38 \mathrm{a}$ \\
\hline Massa seca foliar (g) & $0.77 \pm 0.34 \mathrm{a}$ & $0.58 \pm 0.14 \mathrm{a}$ \\
\hline \multicolumn{3}{|l|}{ Pera glabrata } \\
\hline Altura da planta $(\mathrm{m})$ & $4.83 \pm 0.73 \mathrm{a}$ & $4.16 \pm 0.87 \mathrm{a}$ \\
\hline Diâmetro do caule $(\mathrm{cm})$ & $30.6 \pm 7.41 \mathrm{a}$ & $32.10 \pm 5.58 \mathrm{a}$ \\
\hline Comprimento do elemento de vaso (mm) & $869.01 \pm 137.22 \mathrm{a}$ & $768.36 \pm 128.13 \mathrm{a}$ \\
\hline Diâmetro tangencial do vaso $(\mathrm{mm})$ & $103.98 \pm 12.16 \mathrm{a}$ & $104.62 \pm 11.14 \mathrm{a}$ \\
\hline Frequência de vasos $\left(\mathrm{n} .{ }^{\circ} / \mathrm{mm}^{2}\right)$ & $9.61 \pm 4.34 \mathrm{a}$ & $7.98 \pm 3.57 \mathrm{a}$ \\
\hline Comprimento foliar (mm) & $60.95 \pm 7.09 \mathrm{~b}$ & $89.83 \pm 11.99 \mathrm{a}$ \\
\hline Largura foliar (mm) & $28.22 \pm 3.32 \mathrm{~b}$ & $39.02 \pm 6.29 \mathrm{a}$ \\
\hline Área foliar $\left(\mathrm{cm}^{2}\right)$ & $10.03 \pm 1.95 \mathrm{~b}$ & $21.58 \pm 5.85 \mathrm{a}$ \\
\hline Área específica foliar (cm²/g) & $63.71 \pm 16.48 b$ & $75.64 \pm 17.38 \mathrm{a}$ \\
\hline Massa seca foliar (g) & $0.18 \pm 0.9 \mathrm{~b}$ & $0.30 \pm 0.10 \mathrm{a}$ \\
\hline \multicolumn{3}{|l|}{ Ocotea pulchella } \\
\hline Altura da planta (m) & $4.44 \pm 0.99 \mathrm{a}$ & $4.27 \pm 0.69 \mathrm{a}$ \\
\hline Diâmetro do caule $(\mathrm{cm})$ & $34.6 \pm 7.38 \mathrm{a}$ & $32.4 \pm 4.42 \mathrm{a}$ \\
\hline Comprimento do elemento de vaso (mm) & $606.99 \pm 101.01 \mathrm{a}$ & $644.69 \pm 101.16 \mathrm{a}$ \\
\hline Diâmetro tangencial do vaso (mm) & $112.33 \pm 23.19 \mathrm{a}$ & $104.94 \pm 19.98 \mathrm{a}$ \\
\hline Frequência de vasos $\left(\mathrm{n} .{ }^{\circ} / \mathrm{mm}^{2}\right)$ & $17.98 \pm 6.92 \mathrm{a}$ & $15.56 \pm 7.68 \mathrm{a}$ \\
\hline Comprimento foliar (mm) & $51.00 \pm 7.04 \mathrm{a}$ & $51.86 \pm 6.38 \mathrm{a}$ \\
\hline Largura foliar (mm) & $19.52 \pm 2.34 \mathrm{a}$ & $18.62 \pm 2.74 \mathrm{a}$ \\
\hline Área foliar $\left(\mathrm{cm}^{2}\right)$ & $6.13 \pm 1.42 \mathrm{a}$ & $5.95 \pm 1.39 \mathrm{a}$ \\
\hline Área específica foliar (cm²/g) & $65.47 \pm 8.98 b$ & $71.16 \pm 11.99 \mathrm{a}$ \\
\hline Massa seca foliar $(\mathrm{g})$ & $0.09 \pm 0.02 \mathrm{a}$ & $0.08 \pm 0.01 \mathrm{a}$ \\
\hline \multicolumn{3}{|l|}{ Myrsine venosa } \\
\hline Altura da planta (m) & $4.96 \pm 0.83 \mathrm{a}$ & $4.44 \pm 0.60 \mathrm{a}$ \\
\hline Diâmetro do caule $(\mathrm{cm})$ & $23.6 \pm 4.45 \mathrm{~b}$ & $29.0 \pm 4.10 \mathrm{a}$ \\
\hline Comprimento do elemento de vaso (mm) & $614.69 \pm 97.34 \mathrm{a}$ & $627.73 \pm 95.02 \mathrm{a}$ \\
\hline Diâmetro tangencial do vaso $(\mathrm{mm})$ & $60.76 \pm 9.06 \mathrm{a}$ & $64.01 \pm 12.17 \mathrm{a}$ \\
\hline Frequência de vasos $\left(\mathrm{n} .{ }^{\circ} / \mathrm{mm}^{2}\right)$ & $43.01 \pm 8.48 \mathrm{a}$ & $38.86 \pm 7.25 \mathrm{~b}$ \\
\hline Comprimento foliar (mm) & $101.52 \pm 13.44 b$ & $115.50 \pm 16.28 \mathrm{a}$ \\
\hline Largura foliar (mm) & $44.75 \pm 5.85 \mathrm{a}$ & $44.68 \pm 7.89 \mathrm{a}$ \\
\hline Área foliar $\left(\mathrm{cm}^{2}\right)$ & $26.74 \pm 6.19 b$ & $30.30 \pm 8.26 \mathrm{a}$ \\
\hline Área específica foliar (cm²/g) & $50.78 \pm 7.42 \mathrm{~b}$ & $54.10 \pm 9.48 \mathrm{a}$ \\
\hline Massa seca foliar (g) & $0.53 \pm 0.13 b$ & $0.57 \pm 0.15 \mathrm{a}$ \\
\hline \multicolumn{3}{|l|}{ Ternstroemia brasiliensis } \\
\hline Altura da planta $(\mathrm{m})$ & $4.49 \pm 0.87 \mathrm{a}$ & $4.73 \pm 0.61 \mathrm{a}$ \\
\hline Diâmetro do caule $(\mathrm{cm})$ & $36.0 \pm 5.84 \mathrm{a}$ & $29.9 \pm 4.20 \mathrm{~b}$ \\
\hline Comprimento do elemento de vaso ( $\square \mathrm{m})$ & $1359.57 \pm 270.02 \mathrm{a}$ & $1273.46 \pm 225.73 \mathrm{a}$ \\
\hline Diâmetro tangencial do vaso ( $\square \mathrm{m})$ & $55.94 \pm 10.88 \mathrm{a}$ & $52.82 \pm 11.48 \mathrm{a}$ \\
\hline Frequência de vasos $\left(\mathrm{n} . \mathrm{o} / \mathrm{mm}^{2}\right)$ & $97.33 \pm 7.36 \mathrm{a}$ & $86.00 \pm 13.23 \mathrm{a}$ \\
\hline Comprimento foliar (mm) & $65.93 \pm 9.12 \mathrm{a}$ & $64.33 \pm 7.46 \mathrm{a}$ \\
\hline Largura foliar (mm) & $25.49 \pm 3.55 \mathrm{a}$ & $19.98 \pm 3.72 b$ \\
\hline Área foliar $\left(\mathrm{cm}^{2}\right)$ & $10.3 \pm 1.96 \mathrm{a}$ & $7.3 \pm 1.84 b$ \\
\hline Área específica foliar $\left(\mathrm{cm}^{2} / \mathrm{g}\right)$ & $40.03 \pm 11.55 b$ & $55.25 \pm 9.12 \mathrm{a}$ \\
\hline Massa seca foliar $(\mathrm{g})$ & $0.27 \pm 0.07 \mathrm{a}$ & $0.13 \pm 0.05 \mathrm{~b}$ \\
\hline
\end{tabular}


Tabela 3. Índice de plasticidade fenotípica (IPF) para as variáveis anatômicas e morfológicas das espécies.

\begin{tabular}{|c|c|c|c|c|c|}
\hline \multirow{2}{*}{ Espécie / Variável } & \multicolumn{5}{|c|}{ IPF das espécies } \\
\hline & C. criuva & P. glabrata & O. pulchella & M. venosa & T. brasiliensis \\
\hline Altura da planta $(\mathrm{m})$ & 0.39 & 0.50 & 0.51 & 0.41 & 0.42 \\
\hline Diâmetro do caule (cm) & 0.38 & 0.59 & 0.51 & 0.37 & 0.52 \\
\hline Comprimento do vaso (mm) & 0.37 & 0.43 & 0.5 & 0.46 & 0.49 \\
\hline Diâmetro tangencial do vaso (mm) & 0.64 & 0.33 & 0.59 & 0.54 & 0.73 \\
\hline Frequência de vaso $\left(\mathrm{n} . \% \mathrm{~mm}^{2}\right)$ & 0.79 & 0.95 & 0.91 & 0.71 & 0.61 \\
\hline Comprimento foliar (mm) & 0.52 & 0.54 & 0.64 & 0.57 & 0.51 \\
\hline Largura foliar (mm) & 0.77 & 0.59 & 0.48 & 0.76 & 0.70 \\
\hline Área foliar $\left(\mathrm{cm}^{2}\right)$ & 0.88 & 0.82 & 0.69 & 0.74 & 0.77 \\
\hline Área específica foliar $\left(\mathrm{cm}^{2} / \mathrm{g}\right)$ & 0.73 & 0.87 & 0.68 & 0.75 & 0.80 \\
\hline Massa seca foliar $(\mathrm{g})$ & 0.87 & 0.85 & 0.75 & 0.79 & 0.89 \\
\hline
\end{tabular}

plasticidade fenotípica ocorreram no atributo de massa seca foliar, exceto em C. criuva, que foi mais plástica em área foliar, e P.glabrata, que apresentou maior plasticidade para AEF. Conforme afirmam Smith et al. (1997), a folha, por ser o órgão primário de síntese, tem maior plasticidade e é o que mais responde estruturalmente. Em ambientes mais restritivos em recursos, espera-se que as plantas possuam áreas foliares menores (Larcher 2000), o que não se observa para todas as espécies neste estudo. Assim, sugere-se que em solos nutricionalmente pobres as plantas apresentem maior investimento em tecidos mecânicos uma vez que estes, ao terem maior longevidade, reduzem o custo de produção de novas folhas. Em consquencia, os elevados valores de massa seca, associados à redução da área foliar, resultam em menores valores de AEF nas plantas sob maior restrição de nutrientes (Hodgson et al. 2011, Todorovisky et al. 2015). A AEF pode estar diretamente relacionada ao conteúdo de água nas folhas e à presença de tecidos lignificados, e ambas as situações são estratégias de economia de recursos, sendo água e carbono, respectivamente (Vendramini et al. 2002).

$\mathrm{O}$ atributo anatômico de caule com a maior plasticidade foi a frequência dos vasos (Tab. 3), o que reflete as estratégias das plantas para maximizar a condutividade hidráulica em ambientes comumente caracterizados pela escassez de água, exceto em T. brasiliensis, que foi mais plástica com relação ao diâmetro do elemento de vaso, também uma característica para melhorar a eficiência hidráulica. No entanto, os atributos anatômicos da madeira apresentaram, de modo geral, menor IPF, evidenciando a maior herdabilidade de caracteres ou maior grau de resposta às variações edáficas associado a outros módulos das plantas de acordo com o tipo de fator ou recurso mais influente sobre as populações (Kroon et al. 2005). No presente estudo, os módulos foliares demonstraram maior IPF em virtude da variação na disponibilidade de nutrientes, associada provavelmente à variação luminosa que ocorre nos ambientes de restinga, influenciando diretamente ajustes na morfoanatomia foliar (Kroon et al. 2005). Além disso, o menor IPF em atributos anatômicos da madeira indica condições muito similares de aproveitamento hídrico para tais espécies.

\section{CONCLUSÃO}

Evidenciamos que as espécies estudadas apresentaram certa convergência nas respostas anatômicas do lenho e morfológicas foliares em cada área. De um modo geral, os atributos anatômicos do lenho mostraram-se canalizados entre as populações de cada espécie, mas apresentaram uma tendência de aumento na frequência, diâmetro e comprimeto dos vasos nas populações de São Francisco do Sul, possivelmente em detrimento do maior aporte hídrico neste local. Por outro lado, os atributos morfológicos evidenciaram maior expansão foliar e investimento em tecido fotossintético nos indivíduos de Barra do Sul, o que pode estar relacionado à melhor condição edáfica nesta área, representada pela baixa acidez e balanço entre nutrientes no solo. A restinga de São Francisco do Sul parece ser um ambiente mais restritivo quando comparado com a restinga da Barra do Sul, ao passo que os menores valores de AEF refletem a maior economia de recursos e o investimento em produção foliar. O reduzido IPF para os atributos do lenho evidenciou uma condição hídrica similar entre as áreas e a consequente convergência anatômica entre as espécies estudadas. Sendo assim, sugere-se que o filtro ambiental é decorrente da qualidade nutricional dos solos das restingas.

\section{REFERÊNCIAS}

Amaral Filho, J.P.R, Fornasieri Filho, D., Farinelli, R. \& Barbosa, J.C. 2005. Espaçamento, densidade populacional e adubação nitrogenada na cultura do milho. Revista Brasileira de Ciência do Solo 29(3):467473.

Anjos Silva, L. 2010. Plasticidade e aclimatação foliar à irradiância em espécies da floresta atlântica. Tese 120f. Universidade Federal de Viçosa, Viçosa.

Araújo, D.S.D. \& Lacerda, L.D. 1987. A natureza das restingas. Ciência Hoje 6(33):42-48.

Baas, P. \& Carlquist, S.A. 1985. Comparison of the ecological wood anatomy of the floras of southern California and Israel. International Associate of Wood Anatomists Journal 6(4):349-353.

Baas, P., Wheeler, E. \& Fahn, A. 1983. Some ecological trends in vessel characters. International Associate of Wood Anatomists Journal 4(2-3): 141-159. 
Barros, C.F., Callado, C.H., Marcon, M.L., Costa, C.G., Cunha, M., Lima, H.R.P. \& Marquete, O. 2001. Madeiras da mata atlântica: anatomia do lenho de espécies ocorrentes nos remanescentes florestais do estado do Rio de Janeiro - Brasil. Instituto de Pesquisas do Jardim Botânico do Rio de Janeiro, Rio de Janeiro. 100 p.

Berlyn, G.P. \& Miksche, J.P. 1976. Botanical microtechnique and cytochemistry. Iowa State University, Ames. 326 p.

Boeger, M.R.T., Espíndola Júnior, A., Macari Júnior, A., Reissmann, C.B., Alves, A.C.A. \& Rickli F. L. 2009. Variação estrutural foliar de espécies medicinais em consórcio com erva-mate, sob diferentes intensidades luminosas. Floresta 31(1):215-225.

Boeger, M.R.T. \& Gluzezak, R.M. 2006. Adaptações estruturais de sete espécies de plantas para as condições ambientais da área de dunas de Santa Catarina, Brasil. Iheringia. Série Botânica 61:73-82.

Boeger, M.R.T, Gluzezak, R.M, Pil, M.W., Goldenberg, R. \& Medri, M. 2008. Variabilidade morfológica foliar de Miconia sellowiana (DC.) Naudin (Melastomataceae) em diferentes fitofisionomias no estado do Paraná. Revista Brasileira de Botânica 31(3):443-452.

Carlquist, S. 1977. Ecological factors in wood evolutions: a floristic approach. American Journal of Botany 64(7):887-896.

Climate-Data. 2014. Available at: http://pt.climate-data.org/location/194495/. Acessed on 10 December 2014.

Crawley, M.J. 2007. The R book. John Wiley and Sons, Chichester. 950 p.

Denardi, L. \& Marchiori, J.N.C. 2005. Anatomia ecológica da madeira de Blepharocalyx salicifolius (H. B. K.) Berg. Ciência Florestal 15(2):119-127.

Dickison, W.C. 2000. Integrative plant anatomy. Academy Press, California. $533 \mathrm{p}$.

EMBRAPA. 1997. Manual de métodos de análise de solo. Centro Nacional de Pesquisa de Solos, Riode Janeiro. 212 p. Dispnível em:https:// www.agencia.cnptia.embrapa.br/Repositorio/Manual+de+Metodos 0 00fzvhotqk02wx5ok0q43a0ram31wtr.pdf. Acessado em 01/02/2016.

Ennos, A.R. 1999. The aerodynamics and hydrodynamics of plants. The Journal of Experimental Biology 202(23):3281-3284.

Faquin, V. 2005. Nutrição mineral de plantas. Monografia 186 f. Universidade Federal de Lavras, Lavras.

Franklin, G.L. 1945. Preparation of thin sections of synthetic resins and wood-resin composites, and a new macerating method for wood. Nature 155(3924):51

Gondim, A.R.O., Puiatti, M., Ventrella, M.C. \& Cecon, P.R. 2008. Plasticidade anatômica da folha de taro cultivado sob diferentes condições de sombreamento. Bragantia 67(4):1037-1045.

Gratani, L. 2014. Plant phenotypic plasticity in response to environmental factors. Advances in Botany 313:1-17.

Hodgson, J.G., Montserrat-Marti, G., Charles, M., Jones, G., Wilson, P., Shipley, B., Sharafi, M., Cerabolini, B.E.L., Cornelissen, J.H.C., Band, S.R., Bogard, A., Castro-Díez, P., Guerrero-Campo, J., Plamer, C., Pérez-Rontomé, M.C., Carter, G., Hynd, A., Romo-Díez, A., Espuny, L.T. \& Pla, F.H. 2011. Is leaf dry matter content a better predictor of soil fertility than specific leaf area? Annals of Botany 108:1337-1345.

IAWA Committee. 1989. List of microscopic features for hardwood identification. International Association of Wood Anatomists Journal $10(3): 219-332$

IBGE. 2012. Manual técnico da vegetação brasileira. Instituto Brasileiro de Geografia e Estatística, São Paulo. 271 p.

Johansen, D.A. 1940. Plant microtechnique. McGraw-Hill, New York. $423 \mathrm{p}$.

Knie, J.L.W. 2002. Atlas ambiental da região de Joinville, complexo hídrico da Baía da Babitonga. Fundação de Amparo à Tecnologia e ao Meio Ambiente, Deutsch Gesellschaft Für Technische Zusammernarbeit, Joinville. 144 p.

Kraus, J.E. \& Arduin, M. 1997. Manual básico de métodos em morfologia vegetal. Universidade Federal Rural do Rio de Janeiro, Seropédica. 198 p.

Kroon, H., Huber, H., Stuefer, J.F. \& Groenendael, J.N. 2005. A modular concept of phenotypic plasticity in plants. New Phytologist 166:73-82.

Larcher, W. 2000. Ecofisiologia vegetal. RiMa, São Carlos. 531 p.

Leão, Z.M.A.N. \& Dominguez, J.M.L. 2000. Tropical Coast of Brazil. Marine Pollution Bulletin 41:112-122.

Lima, M.A.O., Mielke, M.S., Lavinsky, A.O., França, S., Almeida, A.F.A. $\&$ Gomes, F.P. 2010. Crescimento e plasticidade fenotípica de três espécies arbóreas com uso potencial em sistemas agroflorestais. Scientias Forestalis 38(87):527-534.
Lopes, A.S. \& Guidolin, J.A. 1989. Interpretação de análise de solo - conceitos e aplicações. Associação Nacional para Difusão de Adubos, São Paulo. 50 p.

Luchi, A.E. 2004. Anatomia do lenho de Croton urucurana Baill. (Euphorbiaceae) de solos com diferentes níveis de umidade. Revista Brasileira de Botânica 27(2):271-280.

Melo Júnior, J.C.F. \& Boeger, M.R.T. 2015. Riqueza, estrutura e interações edáficas em um gradiente de restinga do Parque Estadual do Acaraí, estado de Santa Catarina, Brasil. Hoehnea 42(2):207-232.

. 2016. Leaf traits and plastic potential of plant species in a lightedaphic gradient from a restinga in southern Brazil. Acta Biológica Colombiana 21:51-62.

Melo Júnior, J.F.C., Bona, C. \& Ceccantini, G. 2012. Anatomia foliar de Copaifera langsdorffii Desf. (Leguminosae): interpretações ecológicas em diferentes condições edáficas de Cerrado. Biotemas 25(4):29-36.

Miguel, P.S.B., Gomes, F.T., Rocha, W.S.D., Martins, C.E., Carvalho, C.A. \& Oliveira, A.V. 2010. Efeitos tóxicos do alumínio no crescimento das plantas: mecanismos de tolerância, sintomas, efeitos fisiológicos, bioquímicos e controles genéticos. CES Revista 24:13-29.

Noble, A.D., Gillman, G.P. \& Ruaysoongnern, S.A. 2000. Cation exchange index for assessing degradation of acid soil by further acidification under permanent agriculture in the tropics. European Journal of Soil Science 51(2):233-243.

Paiva, J.G.A., Fank-De-Carvalho, S.M., Magalhães, M.P. \& Graciano-Ribeiro, D. 2006. Verniz vitral $500^{\circledR}$ : uma alternativa de meio de montagem economicamente viável. Acta Botanica Brasilica 20(2):257-264.

Poorter, H. \& Garnier, E. 1999. Ecological significance of inherent variation in relative growth rate and its components. In Handbook of functional plant ecology (F. Pugnaire \& F. Valladares, eds.). CRC Press, New York, p. 81-120.

Ré-Jorge, L. 2007. Plasticidade fenotípica e herbivoria na uvira, Guapira opposita (Nyctaginaceae) em ambiente de duna e restinga. In Workshop de Prática em Pesquisa em Ecologia da Mata Atlântica. Universidade de São Paulo, São Paulo, p. 1-3.

Ronquim, C.C. 2010. Conceitos de fertilidade do solo e manejo adequado para as regiões tropicais. Embrapa, Campinas. 27 p.

Rosado, B.H.P. \& Mattos, E.A. 2010. Interspecific variation of functional traits in a CAM-tree dominated sandy coastal plain. Journal of Vegetable Science 21:43-54.

Sabbi, L.B.C., Ângelo, A.C. \& Boeger, M.R.T. 2010. Influência da luminosidade nos aspectos morfoanatômicos e fisiológicos de folhas de Schinus terebinthifolius Raddi (Anacardiaceae) implantadas em duas áreas com diferentes graus de sucessão, nas margens do Reservatório Iraí, Paraná, Brasil. Iheringia. Série Botânica 65(2):171181.

Santos, M.L.M., Santos, H.G., Agilo, M.L.D., Souza, J.R.S. \& Godoy, E.G. 2013. Calendário de solos. Embrapa Solos, Rio de Janeiro. 16 p.

SBCS. 2004. Manual de adubação e calagem para os estados do Rio Grande do Sul e de Santa Catarina. Sociedade Brasileira de Ciência do Solo, Porto Alegre. 400 p.

Scarano, F.R. 2002. Structure, function and floristic relantioships of plants communities in stressful habitats marginal to Brazilian Atlantic Rainforest. Annals of Botany 90:517-524.

Silva, K.R., Melo Júnior, J.C.F. \& Boeger, M.R.T. 2016. Variações fenotípicas em Andira fraxinifolia Benth. (Fabaceae) em duas fitofisionomias de restinga. Hoehnea 43(2):229-237.

Smith, W.K., Volgelmann, T.C., Delucia, E.H., Bell, D.T. \& Shepherd, K.A. 1997. Leaf form and photosynthesis: do leaf structure and orientation interact to regulate internal light and carbon dioxide?. Bioscience 47(11):785-793.

Sperry, J.S. 2003. Evolution of water transport and xylem structure International Journal of Plant Science 164(3):S115-S127.

Sultan, S.E. 2000. Phenotypic plasticity for plant development, function and life history. Trends in Plant Science 5(12):537-542.

Timm, L.C., Pires, L.F., Roveratti, R., Arthur, R.C.J., Reichard, K., Oliveira, J.C.M., \& Bacchi, O.O.S. 2006. Field spatial and temporal patterns of soil water content and bulk density changes. Scientia Agricola 63(1):55-64.

Todorovisky, E.C.D., Melo Júnior, J.C.F., Amorim, M.W. \& Silva, M.M. 2015. Potencial plástico de Nectandra oppositifolia Nees. (Lauraceae) em fisionomias de floresta ombrófila densa e restinga. Natureza on line 13(12):70-76 
Valladares, F., Sanches-Gomez, D. \& Zavala, M.A. 2006. Quantitative estimation of phenotypic plasticity: bridging the gap between the evolutionary concept and its ecological applications. Journal of Ecology 94(6):1103-1116.

Veloso, C.A.C., Souza, F.R.S., Pereira, W.L.M. \& Tenório, A.R.M. 2001. Relação cálcio, magnésio e potássio sobre a produção de matéria seca de milho. Acta Amazonica 31(2):193-204.

Vendramini, F., Díaz, S., Gurvich, D.E., Wilson, P.J., Thompson, K. \& Hodgson, J.G. 2002. Leaf traits as indicators of resource-use strategy in floras with succulent species. New Phytologist 154:147-157.

White, P.J. 1998. Calcium channels in the plasma membrane of roots cells. Annals of Botany 81(2):173-183.

Witkowski, E.T.F. \& Lamont, B.B. 1991. Leaf specific mass confounds leaf density and thickness. Oecologia 88(4):486-493.

Zamith, L.R. \& Scarano, F.R. 2004. Produção de mudas de espécies das restingas do município do Rio de Janeiro, RJ, Brasil. Acta Botanica Brasilica 18(1):161-176.

Zar, J.H. 1999. Biostatistical analysis. Upper Saddle River, New Jersey. 663 p. 